AperTO - Archivio Istituzionale Open Access dell'Università di Torino

\title{
An algorithm for concurrent estimation of time-varying quantities
}

\section{This is the author's manuscript}

Original Citation:

Availability:

This version is available http://hdl.handle.net/2318/134042

since 2016-01-12T16:32:40Z

Published version:

DOI:10.1088/0957-0233/23/4/045008

Terms of use:

Open Access

Anyone can freely access the full text of works made available as "Open Access". Works made available under a Creative Commons license can be used according to the terms and conditions of said license. Use of all other works requires consent of the right holder (author or publisher) if not exempted from copyright protection by the applicable law. 


\section{(3) \\ UNIVERSITÀ DEGLI STUDI DI TORINO}

This is an author version of the contribution published on:

Questa è la versione dell'autore dell'opera:

Measurement Science and Technology, Vol. 3 No. 4, 2012

The definitive version is available at:

La versione definitiva è disponibile alla URL:

http://iopscience.iop.org/article/10.1088/0957-

0233/23/4/045008/meta;jsessionid=02F499EFCCDE4B6035986B0918B94E1C.ip-10-

40-2-75 


\title{
An algorithm for Concurrent Estimation of Time-Varying Quantities
}

\author{
Giampaolo E D'Errico, Nadir Murru \\ Istituto Nazionale di Ricerca Metrologica (INRIM) \\ Strada delle Cacce 91, 10135-Torino (I) \\ E-mail: g.derrico@inrim.it, nadir.murru@unito.it
}

\begin{abstract}
An original software system for in-process Bayesian estimation is presented with application to a vector of time-varying measurands. The estimation algorithm, mainly based on the Kalman filter technique, is an innovative application to in-process metrology. The programmed strategy, data flow, system/operator interfaces and implemented routines are illustrated and supported by numerical examples. The system performance is demonstrated by reporting and discussing results of simulation trials of metrological interest. The algorithm proves convergent even in severe trials.
\end{abstract}

Keywords: in-process metrology, time-varying measurands, real time estimation, Kalman technique.

PACS numbers: 06.20.Dk, 07.05.Kf

Submitted to: Meas. Sci. Technol. 


\section{Introduction}

In-process metrology deals with real time elaboration of measurands and related uncertainties [1] during a measurement process. The measurand values — the quantities being estimated [2] - can vary with time (e.g., a mobile position). When dynamic systems (e.g., a mechanical plant or a chemical process) are being monitored, measurement data are typically unavailable in advance and their treatment cannot be postponed for batch-processing. Metrological approaches to dynamic systems can be found in recent literature, e.g., [3, 4, 5, 6, 7].

In-process metrology bears relevance also to on-line estimation of unknown parameters for system identification in adaptive control techniques. Optimization criteria lead to estimators based on the principle of minimizing quadratic loss functions [8, 9]. Estimates attained by Kalman filtering are optimal under diverse such criteria, like least-squares (LS) or minimum-mean-square-error (MMSE). LS and MMSE are recognized criteria in orthodox (i.e., non-Bayesian) statistics, which can be thought of in terms of decision theory (DT) too. In fact, DT is also attractive for a comprehensive approach to estimation inclusive of orthodox as well as Bayesian (such as the Kalman filter [10, 11]) techniques.

Kalman filter theory is well established since more than half a century [10], and its practice is developed with application to several fields. For some applications see, e.g.: in-process measurement system, for enhancing the productivity and quality of grinding processes [12]; human-robot interaction [13]; speed estimation of an induction machine [14; electrical motors [15]; position and orientation tracking systems [16]; human limb angle measurement [17]; motion of the carotid artery estimation [18]; estimation of local wind velocity [19]; process tomography [20].

In this perspective, a Bayesian approach to real time estimation was developed, giving rise to a software (SW) [21], where the Kalman filter technique was customized with applicability to coordinate measuring machines (CMMs). In [21] the case of a scalar, time-invariant, quantity was taken into account, aiming at demonstrating (by simulation) the performance of a procedure designed and implemented for real time, simultaneous estimation of measurand value and relevant measurement uncertainty.

Advancing along the same lines, the present research work addresses a more general case-study. Here - subsuming the scalar one as a particular case - estimation target are vectors of time-varying measurands. However, this non trivial generalization entails peculiar complexities to cope with. Two novel different strategies are presented in order to perform estimations of time--varying measurands (in the cases of cyclic or acyclic patterns). In the present paper, the rationale and performance of a SW realized in Matlab $^{\mathrm{TM}} \ddagger$ are presented and discussed with application to such a complex case-study. This is an innovative application of the Kalman filter technique to in-process metrology.

The paper is organized as follows. The next Section 2 focuses on the estimation

$\ddagger$ Identification of commercial products in this paper does not imply recommendation or endorsement, nor does it imply that the products identified are necessarily the best available for the purpose 
problem from both statistical and computational points of view: the Kalman filter foundation on Bayesian statistics roots is set out and technicalities of interest for designed algorithms are detailed. On this basis, Section 3 is devoted to illustrate the implemented strategy $(\S 3.1)$ and to comment the performance of the demonstrator SW realized and tested by simulation ( $\S 3.2)$ : applications of metrological interest are examplified and discussed. Section 4 closes the paper pointing out current results and outlining future prospects.

\section{From Kalman back to Gauss and Bayes}

In this section, those formulae only needed to clarify the relationship of this technique with Bayesian estimation are focused from the point of view of measurement science, with particular attention to measurand and related uncertainty estimation, according to metrological guidelines [1].

The route from Gauss to Kalman can be found in [11]; in the following, the route is reversed from Kalman to Bayes. For a deeper insight into the filter, the seminal paper by Kalman [10] clearly remains the authoritative reference. As to foundations and technicalities of Bayesian statistics, comprehensive expositions are available in treatises: see, e.g., 22] for estimation techniques also related to DT.

In this sense, the Kalman filter connotes an estimation technique with applicability to dynamic processes modeled by discrete linear equations (non-linearity issues are not dealt with in this research work). With application to vectorial quantities, the process state at the step $k(0 \leq k \leq L$, discrete time with maximum value $L$ determined by a stopping criterion) is denoted by $\mathbf{x}_{k}$ and its step by step evolution is described by the following formula:

$$
\mathbf{x}_{k+1}=A_{k} \mathbf{x}_{k}+B_{k} \mathbf{u}_{k}+\mathbf{w}_{k},
$$

where $\mathbf{x}_{k}$ (process state), $\mathbf{u}_{k}$ (optional control input), and $\mathbf{w}_{k}$ (white noise) are vectors, and $A_{k}, B_{k}$ are matrices which relate the process state at the step $k+1$ with the $k$-th process state and with the $k$-th control input, respectively.

The (indirect) measurement $\mathbf{z}_{k}$ of $\mathbf{x}_{k}$ is modeled as follows:

$$
\mathbf{z}_{k}=H_{k} \mathbf{x}_{k}+\mathbf{v}_{k}
$$

where $\mathbf{v}_{k}$ is introduced due to the measurement uncertainty and $H_{k}$ relates the (observable) output $\mathbf{z}_{k}$ with the (internal) state $\mathbf{x}_{k}$. In metrology terms, $\mathbf{z}_{k}$ and $\mathbf{x}_{k}$ represent the measured quantity values and the measurand, respectively. In these terms, the model is translated into the context of measurement science: according to the international vocabulary of metrology, definitions of 'measurand' and 'measured quantity value' can be found in [2] (clauses 2.3 and 2.10, respectively). To the purpose of the estimation task, it is assumed that $H_{k}=I$ (where $I$ is the identity matrix) so to directly relate the measured quantity values $\mathbf{z}_{k}$ to the measurand $\mathbf{x}_{k}$. The estimation is obtained by

means of the following equations: 


$$
\begin{gathered}
\mathbf{x}_{0}^{-}=\tilde{\mathbf{x}}_{-1}=\tilde{\mathbf{x}}_{\text {expert }} \\
\tilde{\mathbf{x}}_{k}=\mathbf{x}_{k}^{-}+K_{k}\left(\mathbf{z}_{k}-H_{k} \mathbf{x}_{k}^{-}\right), \quad 0 \leq k \leq L \\
\mathbf{x}_{k}^{-}=A_{k-1} \tilde{\mathbf{x}}_{k-1}+B_{k-1} \mathbf{u}_{k-1}, \quad 1 \leq k \leq L
\end{gathered}
$$

where $K_{k}$ is the Kalman gain matrix and $\tilde{\mathbf{x}}_{-1}$ is a pre-process estimate obtained from an expert judgment. The entries of $K_{k}$ assign suitable weights to $\mathbf{z}_{k}$ and $\mathbf{x}_{k}^{-}$: according to the MMSE criterion these weights are obtained by minimizing the expected value $E\left(\mathbf{e}_{k}{ }^{t} \mathbf{e}_{k}\right)$, where $\mathbf{e}_{k}=\left|\tilde{\mathbf{x}}_{k}-\mathbf{x}_{k}\right|$ and the left-superscript $t$ stands for the transposition operator.

In [21], the Kalman filter has been developed in a metrological context, with application to a single time-invariant measurand (the unknown value of a scalar quantity, whose measurement is subject to uncertainty). In this paper, a more general case-study is taken into account: the estimation targets are vectors of time-varying measurands whose components are supposed uncorrelated so to simplify $K_{k}$ into a diagonal matrix.

In order to make explicit how the entry $(i, i)$ of the gain matrix is determined, reference to a single $i$-th component of the measurand vector is taken into account: in the following, to avoid a sloppy notation the reference to $i$ will be omitted, so that the $i$-th component of $\mathbf{x}_{k}^{-}$and $\mathbf{z}_{k}$ (bold face) will be denoted by (italics) $x_{k}^{-}$and $z_{k}$ respectively.

In fact, the Kalman filter implements a Bayesian estimation method, the roots of which can be disclosed noting first that the presence of $\mathbf{w}_{k}$ and $\mathbf{v}_{k}$ introduces a stochastic effect into the model through equations (1), (2). After that, let $X, Z$ be the stochastic counterpart of $x_{k}^{-}, z_{k}$ respectively and let $f=f_{k}$ represent a probability density function (PDF) at the $k$-th step (the subscript is omitted, unless required in PDF's arguments showing computational details). The system of equations (3), (4), (5) can thus be translated in Bayesian statistics terms as follows.

The Bayes' theorem can be written as

$$
f(X \mid Z)=\frac{f(Z \mid X) f(X)}{\int_{-\infty}^{+\infty} f(Z \mid X) f(X) d X}
$$

where $f(X \mid Z)$ is called the posterior density, $f(X)$ the prior density, and $f(Z \mid X)$ the likelihood (the denominator is simply a normalization factor). Let $f(X)=\mathcal{N}\left(x_{k}^{-}, \tilde{\sigma}_{k-1}^{2}\right)$ and $f(Z \mid X)=\mathcal{N}\left(z_{k}, \sigma^{2}\right)$ be Gaussian PDFs. From (6) follows

$$
f(X \mid Z) \propto f(Z \mid X) f(X)=\mathcal{N}\left(\frac{\sigma^{2} x_{k}^{-}+\tilde{\sigma}_{k-1}^{2} z_{k}}{\sigma^{2}+\tilde{\sigma}_{k-1}^{2}},\left(\frac{1}{\sigma^{2}}+\frac{1}{\tilde{\sigma}_{k-1}^{2}}\right)^{-1}\right)
$$


where either

$$
\left\{\begin{array}{l}
\tilde{\sigma}_{-1}^{2}=\sigma_{-1}^{2}, \quad \tilde{\sigma}_{0}^{2}=\sigma_{0}^{2} \\
\sigma^{2}=\tilde{\sigma}_{k}^{2} \\
\tilde{\sigma}_{k+1}^{2}=\left(\frac{1}{\sigma^{2}}+\frac{1}{\tilde{\sigma}_{k-1}^{2}}\right)^{-1} \quad, 0 \leq k \leq L,
\end{array}\right.
$$

or

$$
\left\{\begin{array}{l}
\tilde{\sigma}_{-1}^{2}=\sigma_{-1}^{2}, \sigma^{2}=\sigma_{0}^{2} \\
\tilde{\sigma}_{k}^{2}=\left(\frac{1}{\sigma^{2}}+\frac{1}{\tilde{\sigma}_{k-1}^{2}}\right)^{-1}, 0 \leq k \leq L
\end{array}\right.
$$

In (7) the standard deviations $\sigma_{-1}, \sigma_{0}$ are initialized - according to an expert judgment, or based on technical specifications — and can be used for type B (in terms of Guide [1]) uncertainty treatment: $\tilde{x}_{-1}$ and $\sigma_{-1}$ represent prior knowledge about the measurand value and its uncertainty, respectively. Moreover, $\sigma_{0}$ translates known metrological characteristics of the calibrated measuring system actually being used in the measurement process: e.g., if the system is a CMM, $\sigma_{0}$ can be derived from the volumetric length measurement uncertainty and the volumetric probing uncertainty declared by the CMM's producer [21]. As to the parameter $\sigma$, its value is either recursively updated or kept constant as in algorithm of equation (7a) or (7b), respectively. This choice is up to an expert operator, whose decision is entered in (7) at the routine initialization: updating $\sigma$ by $\sigma=\tilde{\sigma}_{k}$, reflects an increasing confidence in repeated measurements. (The possibility of such a personalization reflects the fact that the estimation algorithm is actually implementing a metrological customization of the basic Kalman filter; another option might be allowing $\sigma$ to vary according to criteria for possible outliers treatment: however this topic is not a matter of the present paper: see [23].)

By application of equations (4), (5), (6) the posterior PDF is the Gaussian $f(X \mid Z)=\mathcal{N}\left(\tilde{x}_{k}, \tilde{\sigma}_{k+1}^{2}\right)$ or $f(X \mid Z)=\mathcal{N}\left(\tilde{x}_{k}, \tilde{\sigma}_{k}^{2}\right)$ if equation $7 \mathrm{a}$ or $7 \mathrm{~b}$ has been chosen, respectively, and where

$$
\tilde{x}_{k}=\frac{\sigma^{2} x_{k}^{-}+\tilde{\sigma}_{k-1}^{2} z_{k}}{\sigma^{2}+\tilde{\sigma}_{k-1}^{2}}, \quad 0 \leq k \leq L .
$$

The result of equation (8) is the metrological realization of the Kalman based equation (4). Equations (7), (8) are the core ingredients of the recursive estimation model. Final results of this model are the estimates obtained at the last step of the recursion, namely the expected value and the standard deviation of the posterior Gaussian $\operatorname{PDF} f_{L}(X \mid Z)$.

In the next section, the performance of the estimation technique shown in the above described model is demonstrated by a SW developed with application to a variety of measurands. These include time-varying quantities exhibiting diverse patterns. 


\section{Demonstrator software}

The intended goal of the Kalman filtering approach developed in the preceding section is to provide a model for real time estimation. Accordingly, a suite of recursive algorithms has been implemented. Aiming at displaying computational performance, functioning of interface with an expert operator, and input/output flow, a simulation procedure has been coded in Matlab ${ }^{\mathrm{TM}}$ and tested by use of realistic measurement data. This procedure is the demonstrator SW presented and discussed in the next part.

\subsection{SW strategy}

The SW design is depicted in the flow chart of Figure 1. In the flow chart, the recursive algorithm is represented by the routine where solid arrows are used. The interface is composed of an initialization part, including dialogue with an expert operator and default settings; finally, the outputs are displayed in terms of measurands and uncertainties estimates, obtained by using (7), (8) evaluated at the final step.

In the initialization phase, the operator enters the dimension of the process state vector whose components are the quantities being estimated (measurands). Moreover, the patterns of the measurands time--variation must be specified in this phase, in order to select the appropriate matrix $B$ and vector $\mathbf{u}$ in the estimation procedure, see (5).

The possible patterns so far available are: linear time-functions, sawtooth, triangular-, square--, sine-waves, exponential and parabolic shapes. These patterns are identified by assigning proper parameters such as slopes and periods. For simulation purpose, also the recursions number $L$ must be specified (in real-time execution, $L$ derives from actual measurement process conditions). To initialize the algorithm, the following expert-based inputs are required: $\tilde{x}_{-1}-$ see $(3)-, \sigma_{-1}^{2}, \sigma_{0}^{2}-$ see (7). It is important to stress that to the combination of the values of these three inputs is related the criterion leading to the choice of $\sigma^{2}$ assignments, according to (7).

For instance, $\sigma_{0}^{2}<\sigma_{-1}^{2}$ translates into a greater confidence into the measurements process relative to prior measurand estimate and its uncertainty (this is the CMM case as shown [21]). In this situation, updating $\sigma^{2}=\tilde{\sigma}_{k}^{2}$ balances the influence of measured data compared to prior information about the measurand. Otherwise $\left(\sigma_{0}^{2}>\sigma_{-1}^{2}\right)$, the option $\sigma^{2}=\sigma_{0}^{2}$ would privilege prior estimates.

From a computational point of view, it is noteworthy that a concurrent processing of all involved measurands can be obtained by matrix and vector algebra. In the next section, simulations implementing this approach are shown and their performance is discussed. 


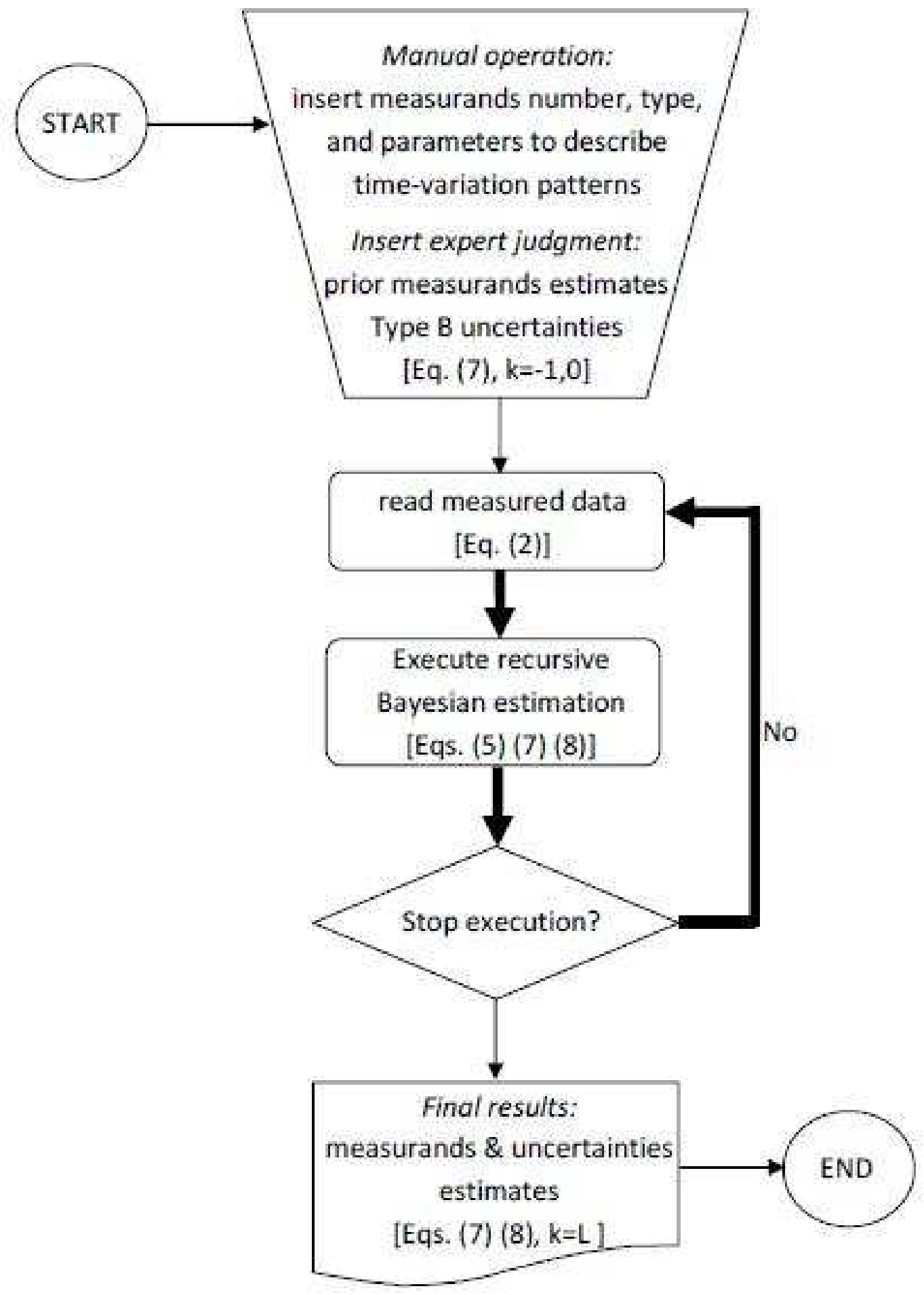

Figure 1. Flow chart: interface and recursive algorithm. 


\subsection{Simulation with discussion}

For simulation purposes, the measurement process model (2) is implemented at each step by using, for each component of 3-dimensional (3D) measurand vector. A normal random function (a white noise) whose standard deviation (SD) is the SD of the corresponding component of $\mathbf{v}_{k}$ (this SD is a descriptor of measurements uncertainty). It is assumed that $A_{k}=B_{k}=I$ (where $I$ is the $3 \times 3$ identity matrix), for every $0 \leq k \leq L$.

Next Figure 2, Figure 3, and Figure 4 report the relevant information with discrete time $k$ on the abscissa and on the ordinate (arbitrary units) the measured values (squares), the estimates (crosses), the prior (expert-based) estimate (circle), and the theoretical time evolution of the measurand (solid line).

Selecting the option $\sigma^{2}=\sigma_{0}^{2}$ in $(7 \mathrm{~b})$, simulations exhibit the behaviour shown in Figure 2 and Figure 4, focused on acyclic time-variation patterns. In Figure $2 a$ (linear time-function): $\sigma_{-1}^{2}=0.02, \sigma_{0}^{2}=0.02$, and $\tilde{x}_{-1}=2.448$. In Figure $2 b$ (parabolic shape): $\sigma_{-1}^{2}=0.1, \sigma_{0}^{2}=0.3$, and $\tilde{x}_{-1}=3.516$. In Figure $2 c$ (exponential shape): $\sigma_{-1}^{2}=0.3$, $\sigma_{0}^{2}=0.1$, and $\tilde{x}_{-1}=7.322$. These patterns may represent cases where a measurand is monitored by tracking its time-evolution trajectory. In Figure $2 a$, it can be noted that even if at the beginning $z_{0}$ and $\tilde{x}_{0}$ are very close to each other, after the first step, the estimates $\tilde{x}_{k}$ are always better than corresponding $z_{k}$. In Figure $2 b$, the situation is not so much different, with the exception of $k=2$, when $z_{2}$ is better positioned (this is a random effect) on the theoretical value: in general, as shown, the estimates are more efficient than measurement data. In Figure $2 c$, it is noteworthy that the effect of a bad expert-based estimate (circle) is quickly recovered after few steps (since $k=2$ ).

On metrological premises, repeated measurements improve estimation: in statistical terms, this translates into selecting the updating mechanism $\sigma^{2}=\tilde{\sigma}_{k}^{2}$. Results of different simulations underpin this position. Related performance is demonstrated in Figure 3, with application to periodic patterns, which may represent measurand's time-variation due to fluctuation of environment conditions (e.g., thermal effects on metal specimen dimensions). In Figure $3 a$ (sine-wave): $\sigma_{-1}^{2}=0.5, \sigma_{0}^{2}=0.5$, and $\tilde{x}_{-1}=1.750$. In Figure $3 b$ (square-wave): $\sigma_{-1}^{2}=0.4, \sigma_{0}^{2}=0.2$, and $\tilde{x}_{-1}=11.771$. In Figure $3 c$ (triangular-wave): $\sigma_{-1}^{2}=0.2, \sigma_{0}^{2}=0.3$, and $\tilde{x}_{-1}=2.129$. In Figure $3 a$ the bad expertbased estimate (circle) affects only the first estimate: starting from $k=1$, estimates remain always close to the theoretical time-evolution curve of the measurand. In Figure $3 b$, a similar situation occurs, where $z_{0}$ is better positioned than $\tilde{x}_{-1}$. In this case, choosing $\sigma^{2}=\tilde{\sigma}_{k}^{2}$, the first estimate is ameliorated by the greater accuracy of $z_{0}$. Even after occurrence of a bad measurement (such as at $k=3$ ), the estimation algorithm is quickly convergent (the effect of an unforeseen over-confidence in measurement is efficiently mitigated). In Figure $3 c, z_{0}$ and $\tilde{x}_{0}$ are almost superimposed and successively measurements, estimates, and the theoretical values of the measurand are close to each other.

The metrological improvement provided by estimates is noticeable at a glance from above discussed simulation trials. However, the algorithm convergence can be further 
exhibited after some severe trials conducted with application to the exponential patterns, using (7b). Related results are shown in Figure 4, under conditions $\sigma_{-1}^{2}>>\sigma_{0}^{2}$ (and vice versa), starting from (purposely) inaccurate prior estimates.

In Figure $4 a: \sigma_{-1}^{2}=5, \sigma_{0}^{2}=0.1$, and $\tilde{x}_{-1}=12$. In Figure $4 b: \sigma_{-1}^{2}=0.3, \sigma_{0}^{2}=0.1$, and $\tilde{x}_{-1}=4$. In Figure $4 c: \sigma_{-1}^{2}=0.1, \sigma_{0}^{2}=1$, and $\tilde{x}_{-1}=7.982$. In Figure $4 a$ the inaccurate expert-based estimate (circle) is immediately compensated. Starting from $k=2$ estimates remain always (except for $k=3$ ) close to the theoretical time-evolution of the measurand. This is due to the large $\sigma_{-1}^{2}$ value that entails a greater weight (compared to prior estimates) assigned to the measured values. In Figure $4 b$ (where $\sigma_{-1}^{2}$ is close to $\sigma_{0}^{2}$ ), the estimates approach the theoretical values of the measurand since $k=8$ (the convergence is slower if compared to the case in Figure $4 a)$. In Figure $4 c\left(\sigma_{0}^{2}=10 \cdot \sigma_{-1}^{2}\right)$, estimates are able to compensate for inaccuracy in measured values, as an effect of the accurate expert-based estimate.

Further examples of the algorithm convergence are shown in Figure 5, where in each panel $\tilde{x}_{-1}$ represents a very inaccurate expert-based estimate. In Figure $5 a: \sigma_{-1}^{2}=0.3$, $\sigma_{0}^{2}=0.1$, and $\tilde{x}_{-1}=0.2$. In Figure $5 b: \sigma_{-1}^{2}=0.3, \sigma_{0}^{2}=0.1$, and $\tilde{x}_{-1}=20$. In Figure $5 c: \sigma_{-1}^{2}=10, \sigma_{0}^{2}=0.1$, and $\tilde{x}_{-1}=20$. Compared to Figure $5 b$, a faster convergence is obtained in Figure $5 c$ thanks to a greater $\sigma_{-1}^{2}$ value (i.e., a greater confidence in measured values).

Table 1, Table 2, and Table 3 contain data of the simulations previously illustrated by Figure 2, Figure 3, and Figure 4 respectively: columns labeled $z, x$, and $\tilde{x}$ report measured data, theoretical values of the measurands, and estimates, respectively; $\tilde{x}_{\text {expert }}=\tilde{x}_{-1}$ is the initial expert judgment.

Table 1 - Measured $(z)$, theoretical $(x)$, and estimated $(\tilde{x})$ values; from Figure 2: linear (a), parabolic (b), and exponential (c) patterns.

\begin{tabular}{|c|c|c|c|c|c|c|c|c|c|}
\hline \multirow[b]{2}{*}{$k$} & \multicolumn{3}{|c|}{ Fig. 2a: $\tilde{x}_{\text {expert }}=2.448$} & \multicolumn{3}{|c|}{ Fig. 2b: $\tilde{x}_{\text {expert }}=3.516$} & \multicolumn{3}{|c|}{ Fig. 2c: $\tilde{x}_{\text {expert }}=7.322$} \\
\hline & $z$ & $x$ & $\tilde{x}$ & $z$ & $x$ & $\tilde{x}$ & $z$ & $x$ & $\tilde{x}$ \\
\hline 0 & 2.447 & 2.444 & 2.448 & 3.497 & 3.541 & 3.511 & 8.152 & 8.167 & 7.944 \\
\hline 1 & 2.432 & 2.452 & 2.447 & 3.194 & 3.545 & 3.451 & 7.592 & 7.535 & 7.432 \\
\hline 2 & 2.485 & 2.459 & 2.462 & 3.550 & 3.557 & 3.477 & 7.377 & 7.302 & 7.253 \\
\hline 3 & 2.490 & 2.466 & 2.473 & 3.762 & 3.577 & 3.535 & 7.200 & 7.217 & 7.175 \\
\hline 4 & 2.422 & 2.473 & 2.471 & 4.164 & 3.605 & 3.638 & 7.127 & 7.185 & 7.140 \\
\hline 5 & 2.439 & 2.481 & 2.472 & 3.665 & 3.641 & 3.673 & 7.210 & 7.173 & 7.142 \\
\hline 6 & 2.496 & 2.488 & 2.482 & 4.167 & 3.685 & 3.762 & 7.114 & 7.169 & 7.134 \\
\hline 7 & 2.478 & 2.495 & 2.488 & 3.622 & 3.737 & 3.797 & 7.226 & 7.168 & 7.144 \\
\hline 8 & 2.478 & 2.502 & 2.493 & 3.407 & 3.797 & 3.819 & 6.972 & 7.167 & 7.125 \\
\hline 9 & 2.562 & 2.509 & 2.506 & 3.647 & 3.865 & 3.869 & 7.219 & 7.167 & 7.134 \\
\hline
\end{tabular}


Table 2 - Measured $(z)$, theoretical $(x)$, and estimated $(\tilde{x})$ values; from Figure 3: sine (a), square (b), and triangular (c) waves.

\begin{tabular}{|c|c|c|c|c|c|c|c|c|c|}
\hline \multirow[b]{2}{*}{$k$} & \multicolumn{3}{|c|}{ Fig. 3a: $\tilde{x}_{\text {expert }}=1.750$} & \multicolumn{3}{|c|}{ Fig. 3b: $\tilde{x}_{\text {expert }}=11.771$} & \multicolumn{3}{|c|}{ Fig. 3c: $\tilde{x}_{\text {sxpert }}=2.123$} \\
\hline & $z$ & $x$ & $\tilde{x}$ & $z$ & $x$ & $\tilde{x}$ & $z$ & $x$ & $\tilde{x}$ \\
\hline 0 & 2.542 & 2.468 & 2.146 & 12.464 & 12.332 & 12.233 & 2.151 & 1.970 & 2.138 \\
\hline 1 & 2.463 & 3.309 & 2.638 & 11.821 & 12.332 & 11.986 & 3.511 & 3.529 & 3.564 \\
\hline 2 & 3.737 & 3.377 & 3.324 & 12.226 & 12.332 & 12.136 & 4.754 & 5.087 & 4.907 \\
\hline 3 & 3.180 & 2.609 & 2.946 & 12.973 & 12.332 & 12.651 & 4.727 & 4.084 & 4,424 \\
\hline 4 & 2.487 & 1.711 & 2.318 & 11.949 & 11.861 & 12.216 & 2.120 & 2.525 & 2.408 \\
\hline 5 & 2.201 & 1.509 & 2.168 & 11.631 & 11.861 & 11.855 & 3.111 & 2.973 & 3.014 \\
\hline 6 & 1.809 & 2.188 & 2.207 & 12.038 & 11.861 & 11.968 & 4.649 & 4.532 & 4.620 \\
\hline 7 & 3.346 & 3.125 & 3.269 & 11.804 & 11.861 & 11.867 & 5.260 & 4.639 & 5.057 \\
\hline 8 & 3.913 & 3.457 & 3.794 & 12.540 & 12.332 & 12.283 & 2.983 & 3.080 & 3.180 \\
\hline 9 & 2.343 & 2.880 & 2.677 & 12.260 & 12.332 & 12.268 & 2.859 & 2.418 & 2.729 \\
\hline
\end{tabular}

Table 3 - Measured $(z)$, theoretical $(x)$, and estimated $(\tilde{x})$ values; from Figure 4: exponential (a), (b), and (c) patterns.

\begin{tabular}{|c|c|c|c|c|c|c|c|c|c|}
\hline \multirow[b]{2}{*}{$k$} & \multicolumn{3}{|c|}{ Fig. 4a: $\tilde{x}_{\text {expert }}=12.000$} & \multicolumn{3}{|c|}{ Fig. $4 \mathrm{~b}: \tilde{x}_{\text {expert }}=4.000$} & \multicolumn{3}{|c|}{ Fig. 4c: $\tilde{x}_{\text {expert }}=7.982$} \\
\hline & $z$ & $x$ & $\tilde{x}$ & $z$ & $x$ & $\tilde{x}$ & 2 & $x$ & $\tilde{x}$ \\
\hline 0 & 8.152 & 8.167 & 8.227 & 8.152 & 8.167 & 7.114 & 8.704 & 8.167 & 8.048 \\
\hline 1 & 7.592 & 7.535 & 7.594 & 7.592 & 7.535 & 6.958 & 9.369 & 7.535 & 7.578 \\
\hline 2 & 7.377 & 7.302 & 7.367 & 7.377 & 7.302 & 6.921 & 5.043 & 7.302 & 7.169 \\
\hline 3 & 7.200 & 7.217 & 7.261 & 7.200 & 7.217 & 6.919 & 8.079 & 7.217 & 7.154 \\
\hline 4 & 7.127 & 7.185 & 7.209 & 7.127 & 7.185 & 6.933 & 7.504 & 7.185 & 7.148 \\
\hline 5 & 7.210 & 7.173 & 7.199 & 7.210 & 7.173 & 6.967 & 5.866 & 7.173 & 7.057 \\
\hline 6 & 7.114 & 7.169 & 7.184 & 7.114 & 7.169 & 6.983 & 6.736 & 7.169 & 7.034 \\
\hline 7 & 7.226 & 7.168 & 7.188 & 7.226 & 7.168 & 7.011 & 7.510 & 7.168 & 7.059 \\
\hline 8 & 6.972 & 7.167 & 7.163 & 6.972 & 7.167 & 7.006 & 10.745 & 7.167 & 7.253 \\
\hline 9 & 7.219 & 7.167 & 7.168 & 7.219 & 7.167 & 7.027 & 9.936 & 7.167 & 7.387 \\
\hline
\end{tabular}

In each panel of Figure 2, Figure 3, and Figure 4, the expert-based initial estimate is pointed out at the instant $k=-1$ : in measurement process term, $\tilde{x}_{-1}$ is in fact a preprocess estimate. On one hand, an accurate pre-process estimate enhances the tracking performance of the algorithm. On the other hand, the influence of $\tilde{x}_{-1}$ on in-process estimates decays after a few steps, so that the algorithm convergence rate is preserved in the presence of a scarce initial estimate too.

Simulation results demonstrate the accuracy provided by in-process estimates also with application to the acyclic patterns shown in Figure 2 and Figure 4 . Following these patterns, it can be noted that each measured value pertains to a diverse measurand state (i.e., measurements are not repeated in identical conditions). However, the theoretical measurand pattern is narrowly tracked by estimates - provided an 
appropriate assignment of constant value to $\sigma^{2}=\sigma_{0}^{2}$ is given in $(7 \mathrm{~b})$, and that vectors $\mathbf{u}_{k}$ are properly pre-set (i.e., according to valuable prior knowledge of the measurand pattern) in (5).

As regards the control input vectors used in the above simulations, they are initialized as follows:

$$
\mathbf{u}_{0}=(0.007,0.004,-0.632), \quad \mathbf{u}_{0}=(0.841,0,1.558), \quad \mathbf{u}_{0}=(-0.632,-0.632,-0.632)
$$

for Figure 2, Figure 3, and Figure 4, respectively. Moreover, using Eq. (8) the entries of the diagonal Kalman gain matrix $K_{k}$ are given by the vector $\frac{\tilde{\boldsymbol{\sigma}}_{k-1}^{2}}{\boldsymbol{\sigma}^{2}+\tilde{\boldsymbol{\sigma}}_{k-1}^{2}}$ (componentwise division). At the step $k=0$ the Kalman gain matrices $K_{0}$ are

$$
\left(\begin{array}{ccc}
0.500 & 0 & 0 \\
0 & 0.250 & 0 \\
0 & 0 & 0.750
\end{array}\right), \quad\left(\begin{array}{ccc}
0.500 & 0 & 0 \\
0 & 0.667 & 0 \\
0 & 0 & 0.400
\end{array}\right), \quad\left(\begin{array}{ccc}
0.980 & 0 & 0 \\
0 & 0.750 & 0 \\
0 & 0 & 0.090
\end{array}\right)
$$

for simulations in Figure 2, Figure 3, and Figure 4, respectively.

In contrast to those represented in Figure 2 and Figure 4, the measurement processes represented in Figure 3 are repeated in cyclically identical conditions, leading to the choice of updating $\sigma^{2}=\tilde{\sigma}_{k}^{2}$ : measurement uncertainty diminishes step after step according to $7 \mathrm{a}$ ).

The stopping criterion for the recursion termination is given in term of maximum number of steps $k=L$. In a real-time measurement process, when no measured data are available in advance, the estimation can be stopped at any desired recursion step $k \geq 1$. It is worthwhile noting that - given initial values of $\tilde{\sigma}_{-1}^{2}$ and $\tilde{\sigma}_{0}^{2}$, the decreasing rate of $\tilde{\sigma}_{k}^{2}$ is a function of the current value $k$ only - a threshold on $\tilde{\sigma}_{k}^{2}$ is also a suitable stopping criterion of real time applicability.

Recalling that measurands and measured data involved in Figures 2, 3, 4 and in Tables 1, 2, 3 are two 3D vectors, relevant simulation results can be resumed in vector form as follows. Let $\mathbf{X}$ and $\mathbf{Z}$ denote the measurand and measured data vectors, respectively. In compliance with guidelines in [24, the expectation and the standard deviation of the posterior PDF $f_{L}(\mathbf{X} \mid \mathbf{Z})$ are taken as the measurand estimate and the associated standard uncertainty, respectively.

As to Figure 2 (see corresponding panels), the (rounded) components of the vectors:

$$
\tilde{\mathbf{x}}_{9}=(2.506,3.869,7.134), \quad \tilde{\boldsymbol{\sigma}}_{9}=(0.04,0.15,0.10)
$$

are the last step $(L=9)$ estimates and their standard uncertainties, respectively. In the same format, final results of the estimation process illustrated in Figure 3 ( $\tilde{\boldsymbol{\sigma}}_{10}$ obtained after equation (7ap) are

$$
\tilde{\mathbf{x}}_{9}=(2.677,12.336,2.729), \quad \tilde{\boldsymbol{\sigma}}_{10}=(0.06,0.04,0.04) .
$$




\section{Conclusion}

An original SW for Bayesian in-process estimation, with application to a vector of time-varying measurands has been implemented in Matlab ${ }^{\mathrm{TM}}$. This is an innovative customization of the Kalman filter for in-process metrology, aimed at combining type A and type B estimation methods according to [1]. Based on the simulation tests carried out, the following facets in the estimation philosophy and system performance can be highlighted.

- simultaneous estimation is conveniently realized on an ensemble of measurands and related uncertainties, by treating each measurand as the component of a same single vector and by using matrix algebra to perform concurrent computation;

- the estimation process really improves the prior measurands knowledge: as a general rule, attained estimates outperform measured data with respect to the theoretical — in principle unknown - measurand time-evolution;

- the uncertainties associated to the estimates decrease at each step;

- the Kalman filter (used in different fields) is successfully applied to the metrological context: this results — as demonstrated — from appropriate use of Gaussian densities in Bayes rule.

Looking at perspective advancements, extension of the model so far presented and discussed to include correlated measurements is envisaged.

Moreover, the presence of possible outlying observations that may occur during the process is a fascinating topic. In fact, it encounters a lot of long standing problems, even worsening when candidate outlier treatment is challenged by real time specifics. However, improvements of the SW system performance can be realistically expected: in a recent work by the same authors [23], the topic of outliers has been tackled from a fuzzy logic point of view - albeit focused on the estimation of a single, time-independent (though subject to uncertainty in measurement) quantity.

For improvement, the realization of an integrated system for real time estimation of a multiplicity of time-varying quantities, possibly mutually correlated, together with real time treatment of suspected outliers is next research target. 

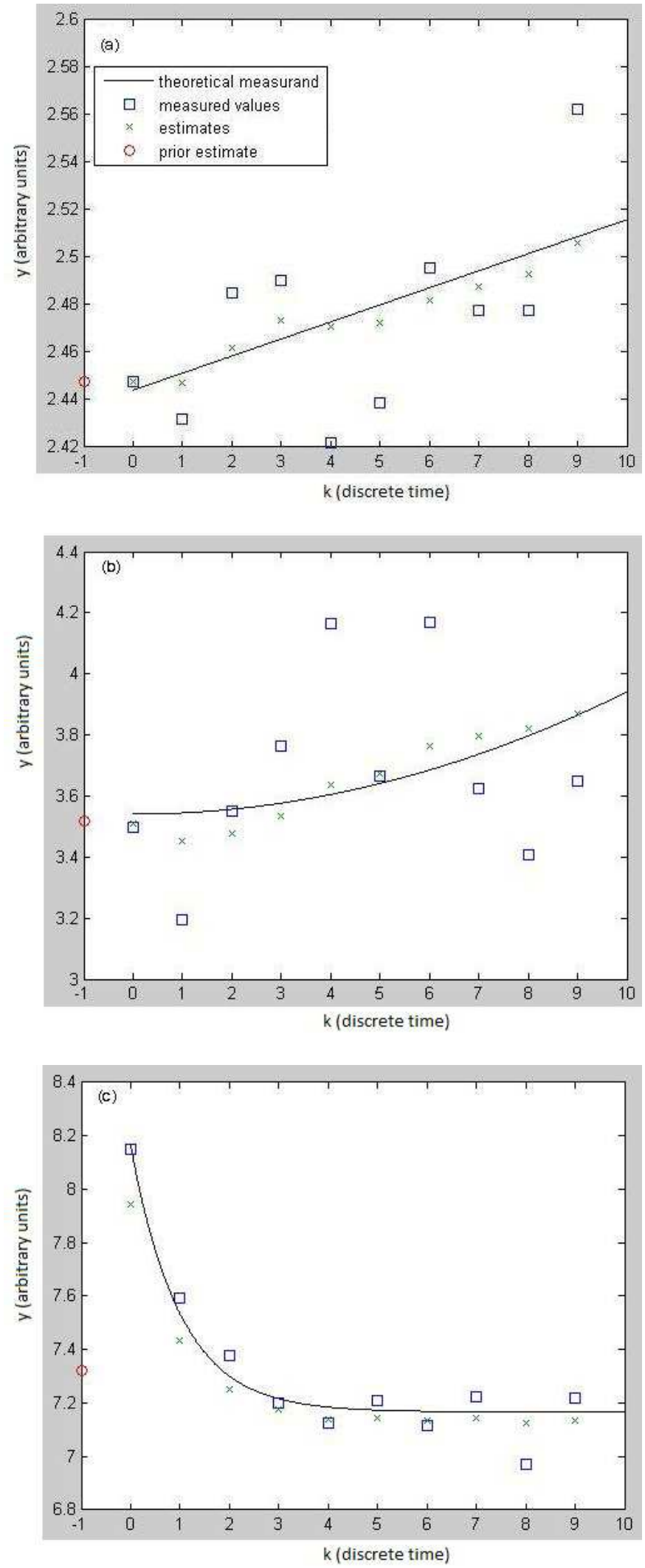

Figure 2 - Acyclic patterns of a 3D vector: simulation results. 

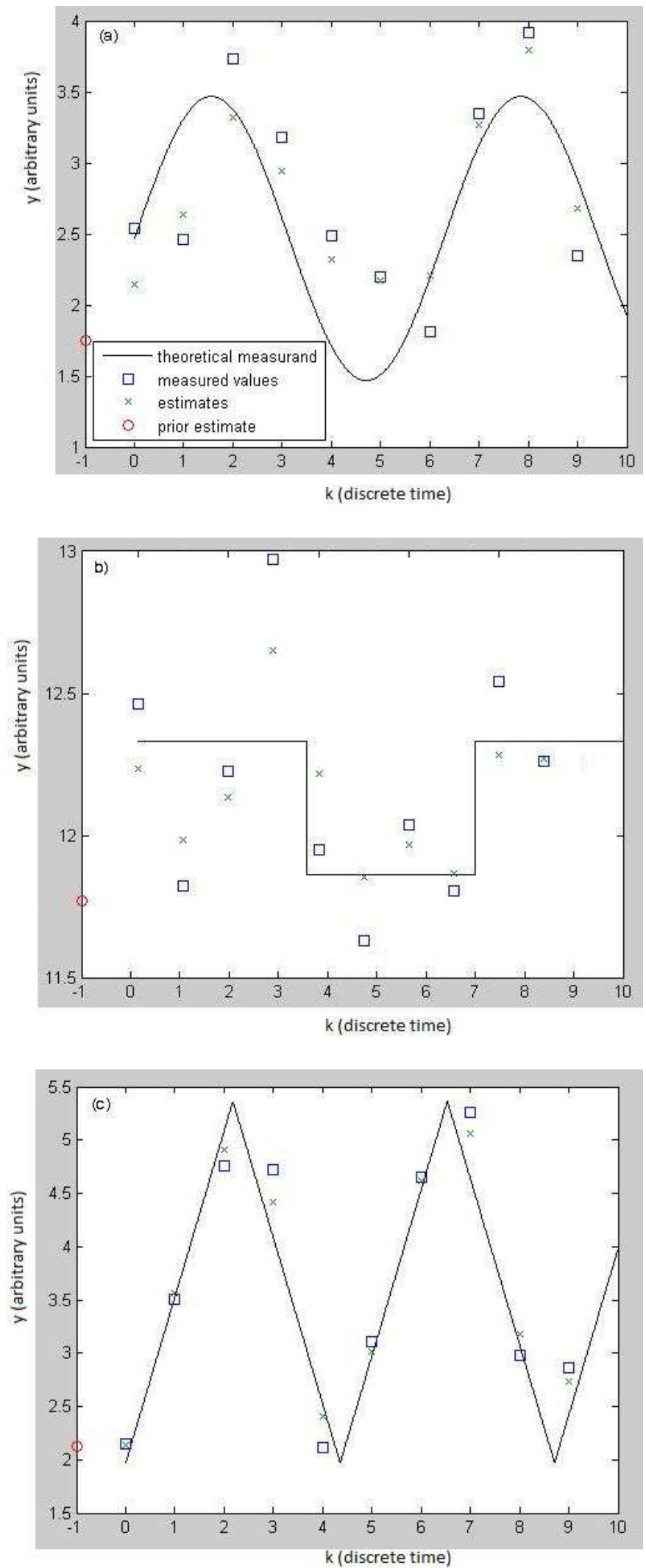

Figure 3 - Cyclic patterns of a 3D vector: simulation results. 

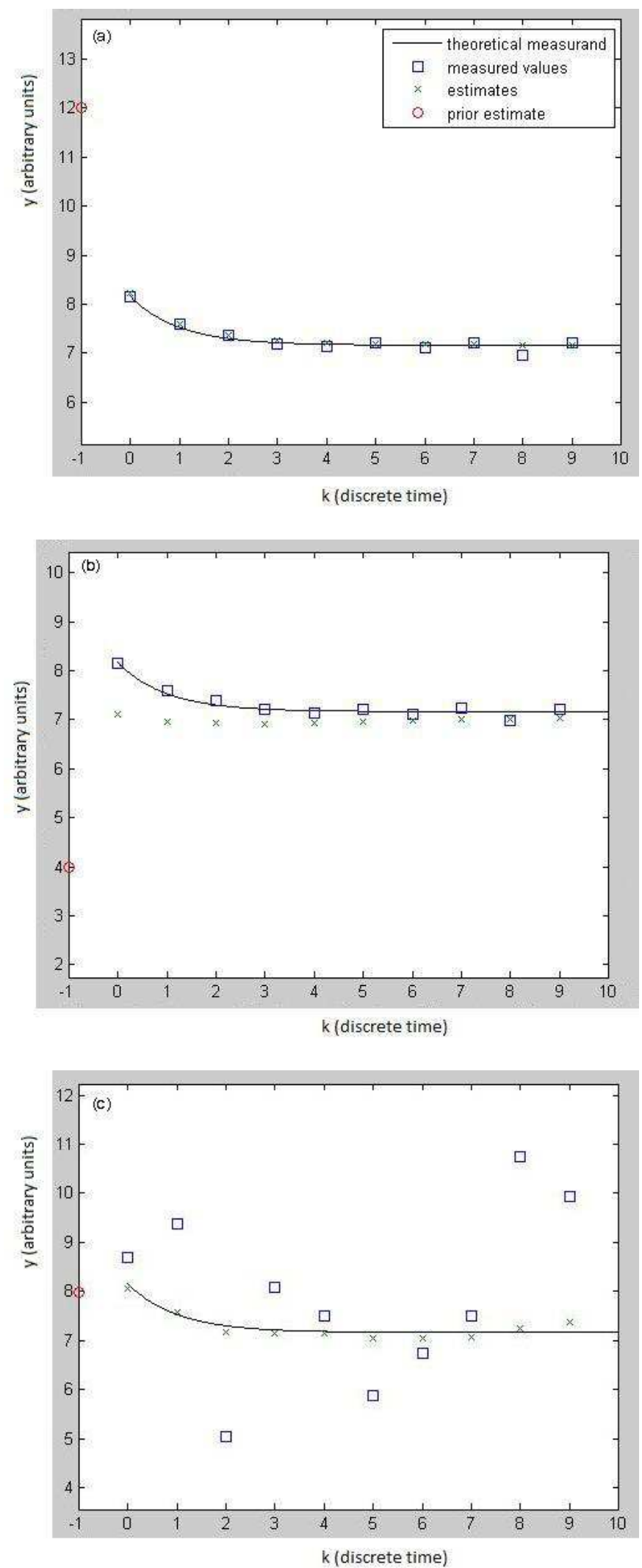

Figure 4 - Performance under severe test conditions. 

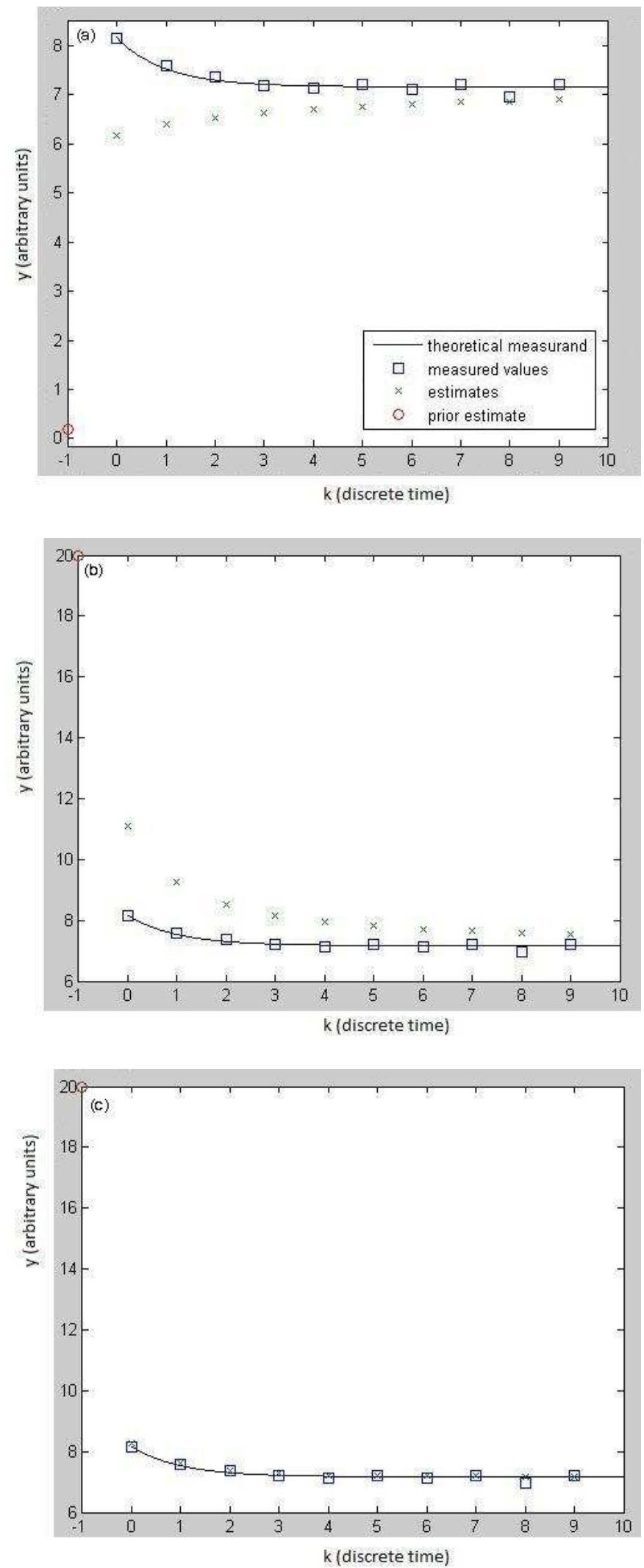

Figure 5 - Convergence after inaccurate prior (expert-based) estimates. 


\section{Nomenclature}

$\tilde{\mathbf{x}}_{k} \quad$ Process estimate vector

$\tilde{\mathbf{x}}_{-1}=\tilde{\mathbf{x}}_{\text {expert }}$ Expert-based prior estimate vector

$\sigma \quad$ Standard deviation (SD), $\tilde{\sigma}_{k}$ SD estimates, $\sigma_{-1}=\tilde{\sigma}_{-1}$ Expert-based prior SD estimate, $\sigma_{0}=\tilde{\sigma}_{0}$ Pre-process SD initialization, $\tilde{\boldsymbol{\sigma}}_{k} \mathrm{SD}$ estimate vector

$\mathbf{u}_{k} \quad$ Control input vector

$\mathbf{v}_{k} \quad$ Measurement noise vector

$\mathbf{w}_{k} \quad$ White noise vector

$\mathbf{x}_{k} \quad$ Process state vector

$\mathbf{x}_{k}^{-} \quad$ Process prior estimate vector

$\mathbf{z}_{k} \quad$ Process measurement vector

$A_{k} \quad$ Transition matrix

$B_{k} \quad$ Control input matrix

$k \quad$ (subscript) Discrete time, iteration step number

$K_{k} \quad$ Kalman gain matrix

$L \quad$ Maximum $k$-value, stopping criterion

\section{References}

[1] BIPM, IEC, IFCC, ISO, IUPAC, IUPAP, and OIML Evaluation of measurement data-guide to the expression of uncertainty in measurement (GUM 1995 with minor corrections) JCGM 100: 2008.

[2] BIPM, IEC, IFCC, ISO, IUPAC, IUPAP, and OIML International Vocabulary of Metrology-Basic and general concepts and associated terms (3rd edn). Guide 99-12:2007

[3] Elster C, Link A, Bruns T 2007 Analysis of dynamic measurements and determination of timedependent measurement uncertainty using a second-order model Meas. Sci. Technol. $183682-$ 87.

[4] Elster C, Link A 2008 Uncertainty evaluation for dynamic measurements modelled by a linear time-invariant system Metrologia 45 464-73.

[5] Hessling J P 2008 Dynamic metrology — an approach to dynamic evaluation of linear timeinvariant measurement systems Meas. Sci. Technol. 19084008.

[6] Hessling J P 2009 A novel method of evaluating dynamic measurement uncertainty utilizing digital filters Meas. Sci. Technol. 20055106.

[7] Hessling J P 2011 Propagation of dynamic measurement uncertainty Meas. Sci. Technol. 22105105.

[8] D'Errico G E 2010 What does mean mean?-A systematic treatment of statistical methods for measurand estimation Measurement 43 504-12.

[9] D'Errico G E 2011 (Unbiased) Variance estimation and uncertainty format Measurement 441128 35.

[10] Kalman R E 1960 A new approach to linear filtering and prediction problems Trans. ASME D, J. Basic Eng. 82 35-45.

[11] Sorenson H W 1970 Least-squares estimation: from Gauss to Kalman IEEE Spectrum $7763-8$.

[12] Lee M H, Bae J L, Yoon K S and Harashima F 2000 Real time and an in-process measuring system for the grinding process cylindrical workpieces using Kalman filtering IEEE Trans. Ind. Electron 476 1326-33. 
[13] Mitsantisuk C, Katsura S and Ohishi K 2009 Kalman-filter-based sensor integration of variable power assist control based on human stiffness estimation IEEE Trans. Ind. Electron. 5610 3897-905.

[14] Hilairet M, Auger F and Berthelot E 2009 Speed and rotor flux estimation of induction machines using a two-stage extended Kalman filter Automatica 458 1819-27.

[15] Salvatore N, Caponio A, Neri F, Stasi S and Cascella G L 2010 Optimization of delayed-state Kalman-filter-based algorithm via differential evolution for sensorless control of induction motors IEEE Trans. Ind. Electron. $571385-94$.

[16] Won S P, Melek W W and Golnaraghi F 2010 A Kalman/particle filter-based position and orientation estimation method using a position sensor/inertial measurement unit hybrid system IEEE Trans. Ind. Electron. 575 1787-98.

[17] Olivares A, Gòrriz J M, Ramìrez J, Olivares G 2011 Accurate human limb angle measurement: sensor fusion through Kalman, least mean squares and recursive least-squares adaptive filtering Meas. Sci. Technol. 22025801.

[18] Gastounioti A, Golemati S, Stoitsis J, Nikita K S 2011 Comparison of Kalman-filter-based approaches for block matching in arterial wall motion analysis from B-mode ultrasound Meas. Sci. Technol. 22114008.

[19] Chan W L, Lee C S, Hsiao F B 2011 Real-time approaches to the estimation of local wind velocity for a fixed-wing unmanned air vehicle Meas. Sci. Technol. 22105203.

[20] Voutilainen A, Lehikoinen A, Vauhkonen M, Kaipio J P 2011 A reduced-order filtering approach for 3D dynamical electrical impedance tomography Meas. Sci. Technol. 22025504.

[21] D'Errico G E 2011 Á la Kalman filtering for metrology tool with application to coordinate measuring machines to appear in IEEE Trans. Ind. Electron. doj 10.1109/TIE.2011.2162212.

[22] Jaynes E T 2003 Probability theory: the logic of science (Cambridge: Cambridge University Press).

[23] D'Errico G E, Murru N 2011 Fuzzy treatment of candidate outliers in measurements to appear in Advances in Fuzzy Systems.

[24] BIPM, IEC, IFCC, ISO, IUPAC, IUPAP, and OIML Evaluation of measurement data-Supplement 1 to the 'Guide to the expression of uncertainty in measurement'-Propagation of distributions using a Monte Carlo method JCGM 101: 2008. 